\title{
On spatial variations of nematic ordering
}

\author{
Ibrahim Fatkullin ${ }^{\text {a }}$, Valeriy Slastikov ${ }^{\mathrm{b}}$ \\ aniversity of Arizona, Department of Mathematics, Tucson, AZ 85721, USA \\ ${ }^{\mathrm{b}}$ University of Bristol, Department of Mathematics, Bristol, BS8 1TW, UK
}

\begin{abstract}
We present a theory of orientational order in nematic liquid crystals which interpolates between several distinct approaches based on the director field (Oseen and Frank), order parameter tensor (Landau and de Gennes), and orientation probability density function (Onsager). As in density-functional theories, the suggested free energy is a functional of spatially-dependent orientation distribution, however, the nonlocal effects are taken into account via phenomenological elastic terms rather than by means of a direct pair-correlation function. In illustration of this approach we consider a simplified model with orientation parameter on a circle and reveal its relation to the complex Ginzburg-Landau theory.
\end{abstract}

Key words: liquid crystals, nematics, spatial patterns PACS: $61.30 . \mathrm{Cz}, 64.70 . \mathrm{Md}, 83.80 . \mathrm{Xz}$

\section{Introduction}

Two basic phenomenological theories addressing spatial variations of orientational order in nematic liquid crystals are the Oseen-Frank $[1,2]$ and Landau-de Gennes [3] theories. The central object in both these theories is a free energy functional; in the former case it is a functional of the field of director (locally-preferred orientation of liquid crystalline molecules), whereas in the latter case it is a functional of the tensor field of order parameter (see the next section for details). Minimizers of the free energy functionals correspond to equilibrium states of liquid crystalline system - a common feature of all free energy-based variational theories.

A microscopic approach to derivation of the free energy was suggested by Onsager [4]. From this point of view the free energy is a functional depending on probability density function of orientations (of liquid crystalline molecules) and may be derived via some cluster or virial expansion (Onsager used the second virial approximation). One of the de- ficiencies of Onsager's theory is insensitiveness to spatial variation of orientation distribution, i.e., the latter is obtained via sampling over all molecules in the system rather than via local, "mesoscopic," sampling. The modern density-functional theories $[5,6]$ (which are in many ways generalizations of Onsager's approach) attempt to solve this issue. Unfortunately, some of their most essential quantities (e.g., the direct pair-correlation function) cannot be readily computed from microscopic principles, so phenomenological approximations still have to be made if one wishes to obtain tangible results.

The goal of this work is to suggest a class of models that may be analyzed and understood analytically (rather than by means of computer simulations). Akin to density-functional method, we base our description on spatially-dependent orientation probability density. However, instead of following the microscopic approach to full extent, we express the order-parameter (or director) field by means of the appropriate averaging, and employ the Oseen-Frank and Landau-de Gennes -type ideas to express the 
elastic part of the free energy. The principal reasons for using this scheme are its simplicity and recent improvements in analytical techniques addressing (spatially-homogeneous) Onsager-type models [7,8]. After describing the general theory we concentrate on a specific model in which the orientation parameter belongs to a circle (rather than a sphere). This simplified model has many properties of the full one and its analysis is quite illustrative mathematically. Additionally, it has an intrinsic relation to complex Ginzburg-Landau-type models and the problem of harmonic mapping into a unit circle. In the last section we discuss various extentions of this work such as incorporation of additional physical features and description of dynamical phenomena in terms of gradient flows generated by the free energy functionals.

\section{Models for nematic liquid crystals}

Let us begin with a brief summary of the theories (mentioned in the introduction) that lay foundation for our work. Since their detailed review is not the goal of this paper, the presentation is considerably simplified (e.g., some terms in the free energy functionals are omitted). Reviews [9-11], or texts such as $[12,3]$ cover the missing details to a great extent.

In what follows we assume the convention that the liquid-crystalline substance is contained in some spatial domain $\Omega \subset \mathbb{R}^{3}$, the corresponding coordinates are denoted by $\boldsymbol{x}, \boldsymbol{y}$, etc. The orientational degree of freedom may be completely characterized by a point on a sphere $\mathbb{S}^{2}$ imbedded in a threedimensional Euclidean space, i.e., $\mathbb{S}^{2}=\left\{s \in \mathbb{R}^{3}\right.$ : $|s|=1\}$. (Strictly speaking if a rod-like molecule is symmetric with respect to inversion its orientation is characterized by point on a two-dimensional projective plane, however it is simpler to use $\mathbb{S}^{2}$ which can be nicely imbedded into $\mathbb{R}^{3}$.)

\subsection{Phenomenological models}

Most phenomenological continuum models describe liquid crystalline systems via macroscopic order-parameter fields. The free energy is considered as a functional of these fields and is constructed using symmetries, qualitative analogies, etc., to possess all essential features known from experiments. Various parameters in such models are tuned so that the theoretical predictions match with experimental observations. In such theories the lack of connection with microscopic (molecular) properties of real liquid crystalline systems is compensated by relative simplicity of mathematical apparatus and good (at least qualitative) agreement with experimental data.

Perhaps the oldest theory that addresses spatial variations of nematic ordering is the Oseen-Frank elastic theory. The state of the system is described via the field of director, $\boldsymbol{n}(\boldsymbol{x})$ - the space-dependent average orientation of liquid crystalline molecules. In essence, it is assumed that the liquid crystalline system is in nematic state and at every macroscopic location $\boldsymbol{x} \in \Omega \subset \mathbb{R}^{3}, \boldsymbol{n}(\boldsymbol{x}) \in \mathbb{S}^{2}$ is well-defined. In its most basic form the free energy functional is given by (the surface terms as well as some other contributions are omitted)

$$
\begin{aligned}
& \mathcal{E}_{\mathrm{OF}}[\boldsymbol{n}]=\frac{1}{2} \int_{\Omega}\left\{\kappa_{11}(\nabla \cdot \boldsymbol{n})^{2}\right. \\
& \left.+\kappa_{22}(\boldsymbol{n} \cdot \nabla \times \boldsymbol{n})^{2}+\kappa_{33}|\boldsymbol{n} \times \nabla \times \boldsymbol{n}|^{2}\right\} \mathrm{d} \boldsymbol{x} .
\end{aligned}
$$

The elastic moduli $\kappa_{\alpha \beta}$ are matched with experiments or microscopic theories $[13,14]$ and may themselves be nontrivial functions of temperature, concentration, etc. (Generally, the indices $\alpha$ and $\beta$ vary from one to six and additional terms may be present in (1). However, due to symmetries only five out of thirty six $\kappa_{\alpha \beta}$ are independent.) The functional (1), even in the reduced form presented here, is intractable analytically, and a simpler model may be obtained under assumption that $\kappa_{11}=\kappa_{22}=\kappa_{33}$. In this case the free energy density becomes $\kappa|\nabla \boldsymbol{n}|^{2} / 2$, which is the usual Dirichlet (elastic) energy. (Needless to say that this simplification is bound to impose additional limitations on the class of phenomena that may be described within this framework.)

Another phenomenological theory is that of Landau and de Gennes. Instead of the director field, here we have the tensor (traceless and symmetric) order-parameter field, $\mathbf{O}(\boldsymbol{x})$. In essence, the components $\mathrm{O}_{\alpha \beta}(\boldsymbol{x})(\alpha, \beta=1,2,3)$ are mesoscopic averages of the products $s_{\alpha} s_{\beta}$ over the liquid crystalline molecules located at macroscopic coordinate $\boldsymbol{x}$. The Landau-de Gennes free energy may be written as

$$
\begin{aligned}
\mathcal{E}_{\mathrm{LdG}}[\mathbf{O}] & =\int_{\Omega}\left\{\frac{\kappa_{1}}{2}|\nabla \mathbf{O}|^{2}+\frac{\kappa_{2}}{2}|\nabla \cdot \mathbf{O}|^{2}\right. \\
+ & \left.a \operatorname{Tr} \mathbf{O}^{2}-b \operatorname{Tr} \mathbf{O}^{3}+c\left[\operatorname{Tr} \mathbf{O}^{2}\right]^{2}\right\} \mathrm{d} \boldsymbol{x} .
\end{aligned}
$$

The gradient terms model elastic interactions whereas the nonlinear terms appear as the result of expansion of the free energy and bear the structure necessary to allow for the isotropic-nematic phase transition. Unlike the Oseen-Frank theory, Landau- 
de Gennes theory also captures variations in the strength of nematic ordering, e.g., one may think of $\operatorname{Tr} \mathbf{O}^{2}$ as a scalar order parameter with $\operatorname{Tr} \mathbf{O}^{2}=0$ corresponding to isotropic state and $\operatorname{Tr} \mathbf{O}^{2} \approx 2 / 3$ corresponding to nematic state. Even though the functional (2) is the simplest functional of the order parameter field (among those that encode all important physical features), its analysis is still very nontrivial as it contains highly nonlinear functions of a tensor field $\mathbf{O}(\boldsymbol{x})$.

\subsection{Onsager model}

Unlike the above theories, the Onsager theory is to a large extent microscopic, i.e., its free energy functional may be (quite rigorously) derived for long rod-like molecules in the limit of small concentration. Onsager's idea is to consider the free energy as a functional of orientation probability density, $\varrho(s)$, and to use asymptotic expansion of this functional with respect to concentration. He assumed that the liquid crystalline molecules may be represented as hard rods of length $\ell$ and diameter $d(\ell \gg d)$ and expanded the free energy (per unit volume) up to second order with respect to concentration, $c$ (this approximation is known as the second virial approximation) obtaining

$$
\begin{aligned}
\mathcal{F}_{\text {Ons }}[\varrho]= & \int_{\mathbb{S}^{2}} \varrho(s) \ln \varrho(s) \mathrm{d} \boldsymbol{s} \\
& +\frac{\gamma}{2} \iint_{\mathbb{S}^{2}} U\left(\boldsymbol{s}, \boldsymbol{s}^{\prime}\right) \varrho(\boldsymbol{s}) \varrho\left(\boldsymbol{s}^{\prime}\right) \mathrm{d} \boldsymbol{s} \mathrm{d} \boldsymbol{s}^{\prime} .
\end{aligned}
$$

(A few terms irrelevant for our study are omitted here.) Unlike parameters in the Oseen-Frank and Landau-de Gennes theories, $\gamma$ is directly related to microscopic properties of liquid crystalline molecules: $\gamma=2 c d \ell^{2}(\mathcal{F}$ is measured in the units of $\left.k_{\mathrm{B}} T\right)$. The interaction potential, $U\left(\boldsymbol{s}, \boldsymbol{s}^{\prime}\right)$, is the so-called second virial coefficient (a multiplicative factor is absorbed into $\gamma$ ). In the original Onsager model

$$
U_{\mathrm{Ons}}\left(s, s^{\prime}\right)=\left[1-\left(s \cdot s^{\prime}\right)^{2}\right]^{1 / 2}=\left|s \times s^{\prime}\right|,
$$

which corresponds to the excluded-volume (hard cores) interaction of liquid-crystalline molecules.

Note also that a phenomenological (mean-field) Maier-Saupe theory [15] produces a free energy functional of Onsager type (3). In their model, however, the interaction (and the parameter $\gamma$ ) is not explicitly derived from the microscopic properties of the system and is rather prescribed as

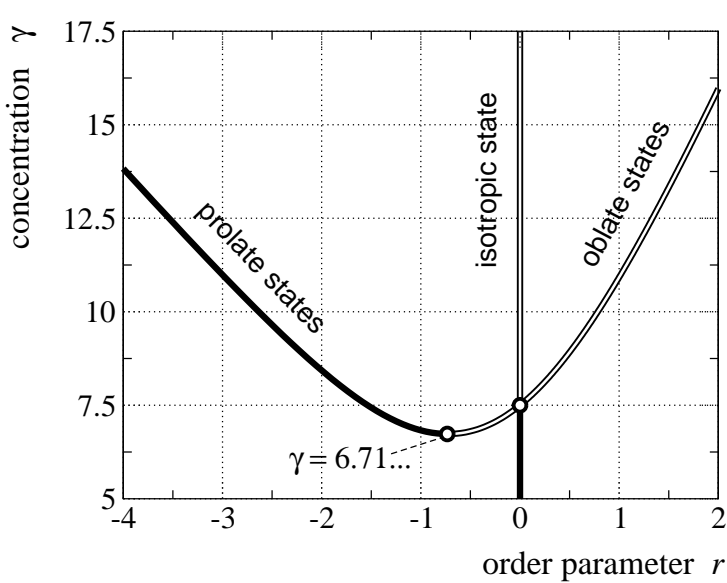

Fig. 1. Isotropic-nematic diagram for the Onsager model (3) with Maier-Saupe interaction (5). The branch $r=0$ corresponds to isotropic phase which is stable when $\gamma<15 / 2$. The curve $\gamma(r)$ obtained from (7) corresponds to nematic states. These states may be prolate $(r<0)$ or oblate $(r>0)$. Oblate states are always unstable, whereas the prolate states may be both stable (left branch) and unstable. At $\gamma \approx 6.76$ (not pictured) the isotropic state becomes "metastable," i.e., it stops being a global minimizer of the free energy functional (remaining a local minimizer while $\gamma<15 / 2$ ).

$$
U_{\mathrm{MS}}\left(s, s^{\prime}\right):=\frac{1}{3}-\left(s \cdot s^{\prime}\right)^{2}
$$

Nevertheless, this interaction has been commonly used since it preserves all essential features of the model and does not suffer from the lack of analyticity as $U_{\text {Ons }}$ does. Even though $\gamma$ in Maier-Saupe approximation is phenomenological, it is still directly proportional to concentration and will be referred to as concentration further on. (Let us also comment that $\gamma$ in Maier-Saupe approximation may be thought of as a factor in multi-pole expansion of the true second virial coefficient, and their model is in this sense a quadrupole approximation.)

In [8] we have shown that all critical points of Onsager functional with Maier-Saupe interaction (also called $\mathcal{P}_{2}$-Onsager model) are given by

$$
\varrho(s)=\mathcal{Z}^{-1} \exp ^{-r(\gamma)\left(3 \cos ^{2} \vartheta-1\right)},
$$

where the constant $\mathcal{Z}$ is obtained from normalization condition, the order parameter $r$ and concentration $\gamma$ are related by

$$
\frac{1}{\gamma}=\frac{1}{6 r} \frac{\mathrm{d}}{\mathrm{d} r} \ln \mathcal{Z}(r) .
$$

See Figure 1 for the corresponding phase (isotropicnematic) diagram.

Onsager's theory captures well the isotropicnematic phase transition and is explicitly solvable, which makes it a perfect starting point for the 
development of more complicated theories that incorporate dependence of the orientation probability density on the spatial coordinate $\boldsymbol{x}$. It became a precursor to modern density-functional theories where the state of the system is described via $\varrho(x, s)$ (or more complex joint probability density functions) and the free energy is written down as a functional of the state. Generally, this functional has a form similar to (3) except the interaction $U$ itself becomes a functional of $\varrho$ (this object is known as direct pair-correlation function). The catch is that no systematic way of computing $U[\varrho]\left(s, s^{\prime}\right)$ is currently known and some phenomenological or ad-hoc approximations still have to be made for obtaining tangible results. (Moreover, the assumption that $U$ may be considered as a functional of $\varrho$ alone does not have to hold and, strictly speaking, the whole hierarchy of joint probability density functions has to be taken into account in a truly complete microscopic theory.)

\subsection{A framework for semi-microscopic models}

Here we present a class of models that in some sense interpolate between Onsager's model and phenomenological models of Oseen-Frank and Landaude Gennes. The idea is to use Onsager's model on "mesoscopic" level and to model the spatial interactions via elastic-like terms as in Oseen-Frank and Landau-de Gennes theories. We base our description on space-dependent orientation probability density function, $\varrho(\boldsymbol{x}, \boldsymbol{s})$, assuming that at every macroscopic location $\boldsymbol{x} \in \Omega$ the latter is well-defined. (Generally, if liquid-crystalline molecules have additional relevant degrees of freedom, $s$ may belong to a more complicated manifold than $\mathbb{S}^{2}$, see Section 4.2 for discussion.)

Such description allows us to recover the orderparameter field of Landau-de Gennes theory: the elements $\mathrm{O}_{\alpha \beta}(\boldsymbol{x})$ may be represented as

$$
\mathrm{O}_{\alpha \beta}(\boldsymbol{x})=\int_{\mathbb{S}^{2}} s_{\alpha} s_{\beta} \varrho(\boldsymbol{x}, \boldsymbol{s}) \mathrm{d} \boldsymbol{s}-\frac{\delta_{\alpha \beta}}{3} .
$$

Recovery of the director field $\boldsymbol{n}(\boldsymbol{x})$ is a more complicated matter and may not even be possible. The reason, of course, is that the description in terms of $\varrho(\boldsymbol{x}, \boldsymbol{s})$ is more complete and incorporates the states for which the director simply cannot be properly defined. Formally, one can find $\boldsymbol{n}(\boldsymbol{x})$ minimizing the distance between $\varrho(x, s)$ and $\delta_{\boldsymbol{n}}(\boldsymbol{s})$ (atomic measure split in equal halves between $\boldsymbol{n} \in \mathbb{S}^{2}$ and the opposite point) in some reasonable metric (e.g., Wasserstein-2 [16]).

Following [17], we suggest to employ a fivedimensional director field defining

$$
\boldsymbol{n}(\boldsymbol{x}):=\int_{\mathbb{S}^{2}} \mathbf{Y}(\boldsymbol{s}) \varrho(\boldsymbol{x}, \boldsymbol{s}) \mathrm{d} \boldsymbol{s},
$$

$\mathbf{Y}(\boldsymbol{s})$ denotes a vector of second-order spherical harmonics $\left[\mathrm{Y}_{2,-2}(\boldsymbol{s}), \ldots, \mathrm{Y}_{2,2}(\boldsymbol{s})\right]$. Note that $\boldsymbol{n}(\boldsymbol{x})$ contains precisely the same information as the Landau-de Gennes order-parameter tensor $\mathbf{O}(\boldsymbol{x})$, since second-order spherical harmonics are the orthogonalized second-order homogeneous polynomials of $s$. The reason to use the averages of spherical harmonics $\mathrm{Y}_{2, m}(\boldsymbol{s})$ rather than the averages of products $s_{\alpha} s_{\beta}$ is that they enjoy a number of nice mathematical properties and are convenient to work with.

Within the framework of our theory the total (bulk) free energy is decomposed into two parts:

$$
\mathcal{E}[\varrho]=\int_{\Omega}\left\{\mathcal{F}_{\mathrm{o}}(\boldsymbol{x})+\mathcal{F}_{\mathrm{e}}(\boldsymbol{x})\right\} \mathrm{d} \boldsymbol{x} .
$$

The first term, $\mathcal{F}_{\mathrm{o}}(\boldsymbol{x})$ is the Onsager-type orientational entropy density (3), which now becomes a function of $\boldsymbol{x}$. The second term, $\mathcal{F}_{\mathrm{e}}(\boldsymbol{x})$ is a phenomenological elastic energy density penalizing variations of orientational ordering. In case of linear elasticity (the energy is quadratic with respect to spatial derivatives of $\boldsymbol{n}$ ) we have

$$
\mathcal{F}_{\mathrm{e}}[\boldsymbol{n}(\boldsymbol{x})]=\frac{1}{2}(\boldsymbol{\partial n}, \hat{\boldsymbol{\kappa}} \boldsymbol{\partial} \boldsymbol{n}),
$$

where the operator $\boldsymbol{\partial}$ has components

$$
\partial_{0}=\partial_{z}, \quad \partial_{ \pm 1}=\left(\partial_{x} \pm \mathrm{i} \partial_{y}\right) / \sqrt{2},
$$

and $\hat{\boldsymbol{\kappa}}$ is a $15 \times 15$ matrix containing elastic moduli. As shown in [17], due to symmetry considerations, only three entries of $\hat{\boldsymbol{\kappa}}$ are independent. Indeed, the products $\partial_{i} n_{j}$ may be combined to form three irreducible tensors $(l=1,2,3 ; m=-l \ldots l)$

$$
[\boldsymbol{\partial} \otimes \boldsymbol{n}]_{m}^{(l)}=\sum_{i=-1}^{1} \sum_{j=-2}^{2}\left(\begin{array}{ccc}
1 & 2 & l \\
i & j & m
\end{array}\right) \partial_{i} n_{j} .
$$

Here the weighting factors are the Clebsch-Gordan coefficients ( $3 j$-symbols). Contracting these tensors we may form three scalar invariants (one for each $l$ )

$$
I^{(l)}=\sum_{m=-l}^{l}\left(\begin{array}{ccc}
l & l & 0 \\
m & -m & 0
\end{array}\right)[\boldsymbol{\partial} \otimes \boldsymbol{n}]_{m}^{(l)}[\boldsymbol{\partial} \otimes \boldsymbol{n}]_{-m}^{(l)} .
$$

Thus the elastic energy (11) may be represented as

$$
\mathcal{F}_{\mathrm{e}}[\boldsymbol{n}(\boldsymbol{x})]=\kappa_{1} I^{(1)}+\kappa_{2} I^{(2)}+\kappa_{3} I^{(2)} .
$$




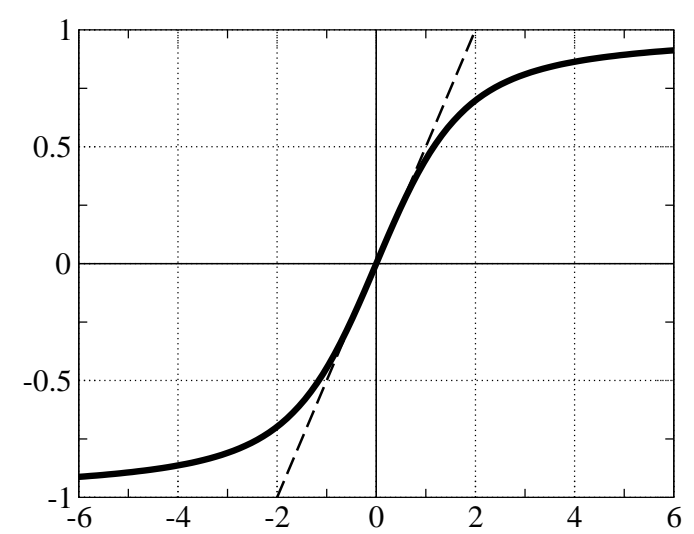

Fig. 2. Graph of $\mathrm{T}(r)=\mathrm{I}_{1}(r) / \mathrm{I}_{0}(r)$. As $r \rightarrow \pm \infty$, $\mathrm{T}(r) \rightarrow \pm 1$. Slope of $\mathrm{T}(r)$ at $r=0$ is $1 / 2$ (the dashed line) reflecting that (18) has nonzero solutions when $\gamma>2$.

Explicit expressions for $I^{(l)}$ may be found in [17]. Some possible generalizations for $\mathcal{F}_{\mathrm{e}}(\boldsymbol{x})$ are discussed in Section 4.1.

Finally, let us mention that in this work we do not analyze the surface effects in such a generality and, instead, illustrate a few possible scenarios on specific examples in the following section.

\section{A two-dimensional $\left(\mathbb{S}^{1}\right)$ model}

In order to illustrate some of the principal features of the suggested class of models, let us study in greater detail one particular example in which the orientation parameter belongs to a unit circle, $\mathbb{S}^{1}$, parameterized by a number in $[0,2 \pi)$. The full threedimensional case (the orientation parameter belongs to a sphere, $\mathbb{S}^{2}$ ) shares lots of similarity with this simplified example and may be analyzed by similar methods. It is, however, more technical and will be considered in a separate paper [18].

As discussed in the previous section, we use an Onsager-type functional (a few irrelevant terms are omitted),

$$
\begin{array}{r}
\mathcal{F}_{\mathrm{o}}[\varrho]:=\int_{0}^{2 \pi} \varrho(\varphi) \ln \varrho(\varphi) \mathrm{d} \varphi \\
-\frac{\gamma}{2} \iint_{0}^{2 \pi} \cos 2\left(\varphi-\varphi^{\prime}\right) \varrho(\varphi) \varrho\left(\varphi^{\prime}\right) \mathrm{d} \varphi \mathrm{d} \varphi^{\prime},
\end{array}
$$

for the orientational free energy density. Note that our interaction, $\cos 2\left(\varphi-\varphi^{\prime}\right)$, is equivalent to the Maier-Saupe interaction (5). As it was shown in [7], all critical points of (16) are given by

$$
\varrho(\varphi)=\frac{\exp \{r \cos 2(\varphi-\psi)\}}{2 \pi \mathrm{I}_{0}(r)},
$$

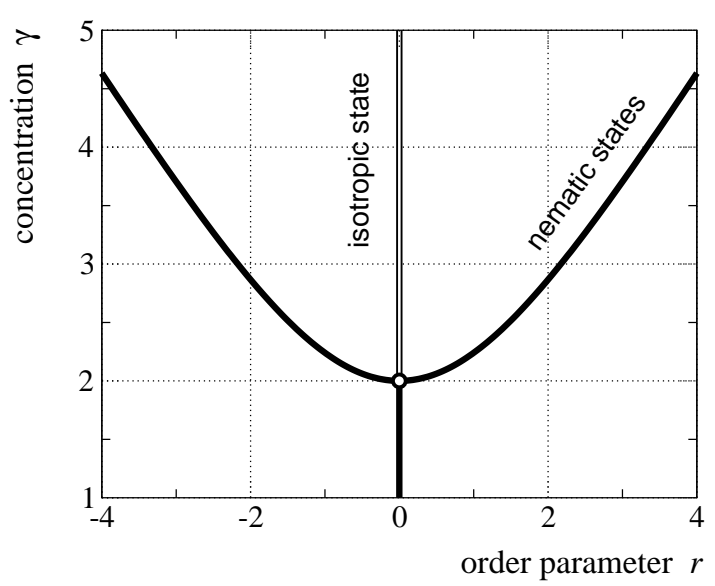

Fig. 3. Isotropic-nematic phase diagram for the free energy functional (16). The vertical line, $r=0$, corresponds to isotropic state which is stable when $\gamma<2$. The curved line is the graph of $\gamma(r)$ obtained from (18); it corresponds to stable nematic states.

where the order parameter $r$ is either zero or is related to concentration $\gamma$ by

$$
\frac{1}{\gamma}=\frac{\mathrm{T}(r)}{r}, \quad \mathrm{~T}(r):=\frac{\mathrm{I}_{1}(r)}{\mathrm{I}_{0}(r)} .
$$

Here $\mathrm{I}_{n}(r)$ are the modified Bessel functions of the first kind; the ratio $\mathrm{I}_{1}(r) / \mathrm{I}_{0}(r)$ appears quite often in what follows, so we designate a special symbol, $\mathrm{T}(r)$, for it (see Figure 2 for the graph of $\mathrm{T}(r)$ ). The graph of functional dependence of $\gamma$ on $r$ is presented in Figure 3. For small concentrations $(\gamma \leq 2)$ $r=0$ is the only critical point (and the global minimizer); when $\gamma>2$ it loses stability and a rotationequivalent family (parameterized by $\psi$ ) (17) of nontrivial global minimizers emerges. Here the major difference with the full $\mathbb{S}^{2}$-model can be spotted: the phase transition in $\mathbb{S}^{1}$-model is of the second order and does not allow for coexistence of stable isotropic and nematic phases (compare Figures 1 and 3). In particular, this implies that the full treatment is necessary in order to describe such phenomena as isotropic-nematic interfaces.

For the elastic free energy density we pick the simplest Dirichlet-type functional

$$
\begin{array}{r}
\mathcal{F}_{\mathrm{e}}[\varrho]:=\frac{\kappa}{2}|\nabla \boldsymbol{n}|^{2} \\
=\frac{\kappa}{2} \iint_{0}^{2 \pi} \cos 2\left(\varphi-\varphi^{\prime}\right) \nabla \varrho(\varphi) \cdot \nabla \varrho\left(\varphi^{\prime}\right) \mathrm{d} \varphi \mathrm{d} \varphi^{\prime} .
\end{array}
$$

The gradient $\nabla$ is with respect to the spatial variable $\boldsymbol{x} \in \Omega$. Here we related the order-parameter field $\boldsymbol{n}(\boldsymbol{x})$ to the spatially-dependent orientation density function $\varrho(\boldsymbol{x}, \varphi)$ via 


$$
\boldsymbol{n}(\boldsymbol{x}):=\int_{0}^{2 \pi} \mathrm{e}^{2 \mathrm{i} \varphi} \varrho(\boldsymbol{x}, \varphi) \mathrm{d} \varphi .
$$

(In $\mathbb{S}^{1}$-model $\boldsymbol{n}$ is two-dimensional rather than fivedimensional as it would be in $\mathbb{S}^{2}$-model, cf (9).) Note that we often treat $\boldsymbol{n}(\boldsymbol{x})$ as a complex scalar field rather than a two-dimensional real vector-field (this simplifies notation and plays no essential role otherwise).

The total free energy is obtained by integrating both contributions, (16) and (19), over the spatial domain $\Omega$ :

$$
\mathcal{E}[\varrho]:=\int_{\Omega}\left\{\mathcal{F}_{\mathrm{o}}[\varrho]+\mathcal{F}_{\mathrm{e}}[\varrho]\right\} \mathrm{d} \boldsymbol{x} .
$$

Now the equilibrium states of the system may be found via minimization of the total free energy (21). This energy, in general, has to be augmented by the boundary terms arising due to interaction of polymers with the container or other surface effects. Typically, the boundary contribution is given by

$$
\mathcal{E}_{\text {bnd }}[\varrho]:=-\int_{\partial \Omega} \boldsymbol{n}(\boldsymbol{x}) \cdot \boldsymbol{u}(\boldsymbol{x}) \mathrm{d} s(\boldsymbol{x}),
$$

where $\boldsymbol{u}(\boldsymbol{x})$ is a boundary potential (it provides the preferred orientation of $\boldsymbol{n}(\boldsymbol{x})$ on the boundary) and $\mathrm{d} s(\boldsymbol{x})$ is the area of the surface element. It is also possible to use a somewhat simplified approach prescribing the boundary conditions for the argument of $\boldsymbol{n}(\boldsymbol{x})$ directly. Such treatment corresponds to an assumption that it is experimentally feasible to control orientation of liquid-crystalline molecules on the boundary of the system.

\subsection{Euler-Lagrange equations}

Let us consider the Euler-Lagrange equations associated with (21). It is convenient to make a change of variables, introducing a potential $\Psi:=\ln \varrho+$ const, choosing the constant so that $\Psi(\varphi)$ integrates to zero over $\varphi \in[0,2 \pi)$. We have

$$
\varrho(\boldsymbol{x}, \varphi)=\mathcal{Z}^{-1}(\boldsymbol{x}) \mathrm{e}^{-\Psi(\boldsymbol{x}, \varphi)},
$$

where the partition integral, $\mathcal{Z}$, is given by

$$
\mathcal{Z}(\boldsymbol{x})=\int_{0}^{2 \pi} \mathrm{e}^{-\Psi(\boldsymbol{x}, \varphi)} \mathrm{d} \varphi .
$$

A straightforward computation shows that EulerLagrange equation for critical points of (21) may be written as

$$
\Psi(\boldsymbol{x}, \varphi)=\int_{0}^{2 \pi} \cos 2\left(\varphi-\varphi^{\prime}\right)[\gamma+\kappa \Delta] \varrho\left(\boldsymbol{x}, \varphi^{\prime}\right) \mathrm{d} \varphi^{\prime} .
$$

(Laplacian, $\Delta$, only acts on the spacial variable $\boldsymbol{x}$.) The boundary contributions into the total free energy supply the boundary conditions, e.g., (22) produces

$$
\kappa \partial_{\perp} \boldsymbol{n}(\boldsymbol{x})=\boldsymbol{u}(\boldsymbol{x}), \quad \boldsymbol{x} \in \partial \Omega,
$$

where $\partial_{\perp}$ denotes the normal derivative. Differentiating both sides of (25) twice with respect to $\varphi$, we immediately find that $\Psi$ satisfies

$$
\Psi_{\varphi \varphi}(\boldsymbol{x}, \varphi)=-4 \Psi(\boldsymbol{x}, \varphi)
$$

which implies that it may be represented as

$$
\Psi(\boldsymbol{x}, \varphi)=r(\boldsymbol{x}) \cos 2[\varphi-\psi(\boldsymbol{x})] .
$$

Substituting this in (24) and integrating we get

$$
\mathcal{Z}(\boldsymbol{x})=2 \pi \mathrm{I}_{0}(r(\boldsymbol{x})),
$$

which implies that critical points of the free energy are given by

$$
\varrho(\boldsymbol{x}, \varphi)=\frac{\exp \{r(\boldsymbol{x}) \cos 2[\varphi-\psi(\boldsymbol{x})]\}}{2 \pi \mathrm{I}_{0}(r(\boldsymbol{x}))} .
$$

It is rather remarkable that in the $\boldsymbol{x}$-dependent model (21) the structure of critical points is very similar to the "homogeneous" case (16): the parameters $r$ and $\psi$ here become the $\boldsymbol{x}$-dependent fields while the structure of the orientation probability density remains the same, of (17). The problem is now reduced to finding $r(\boldsymbol{x})$ and $\psi(\boldsymbol{x})$.

Before proceeding with analysis of the EulerLagrange equation let us reveal a intimate connection of our free energy functional with GinzburgLandau-type functionals.

\subsection{Reduction to a Ginzburg-Landau-type model}

Since we know that all critical points satisfy (30) we may express the free energy as a functional of $\boldsymbol{n}(\boldsymbol{x})$ (or $r(\boldsymbol{x})$ and $\psi(\boldsymbol{x}))$. Integrating (20) using (30) we obtain

$$
\boldsymbol{n}(\boldsymbol{x})=\mathrm{e}^{2 \mathrm{i} \psi(\boldsymbol{x})} \mathrm{T}(r(\boldsymbol{x})) .
$$

Inverting this relation (denoting $n=|\boldsymbol{n}|$ and introducing $\mathrm{A}(n)$, the inverse function of $\mathrm{T}(r)$ ) we may express $r$ as a function of $n$

$$
r=\mathrm{A}(n) .
$$

Now let us integrate over $\varphi$ in (16). The $\varrho \ln \varrho$-part yields

$$
r \mathrm{~T}(r)-\ln \left[2 \pi \mathrm{I}_{0}(r)\right] \text {. }
$$

The interaction part of (16) becomes

$$
-\frac{\gamma}{2} \mathrm{~T}^{2}(r) \text {. }
$$




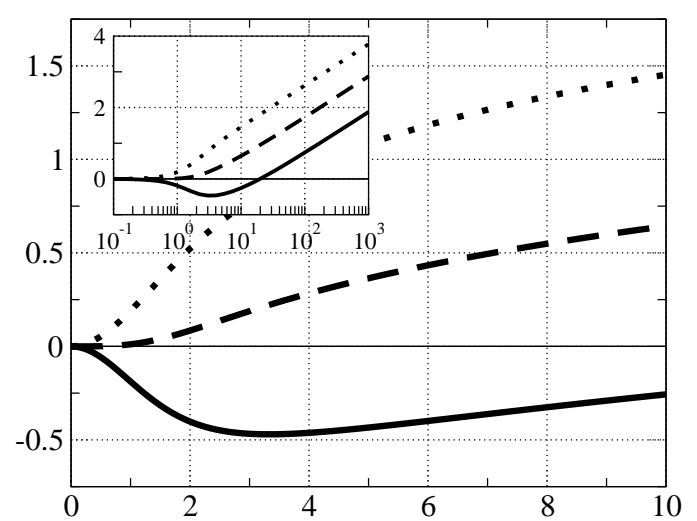

Fig. 4. Graphs of $W(r)$ given by (35). The dashed line corresponds to the critical value, $\gamma=2$; the dotted line - to $\gamma=0.2$; the solid line - to $\gamma=4$. The logarithmic subplot demonstrates that $W(r) \rightarrow \infty$ as $r \rightarrow \infty$ (the growth is logarithmically slow).

Thus we obtain

$$
\begin{aligned}
\mathcal{F}_{\mathrm{o}}[\varrho] & =W(r) \\
=r \mathrm{~T}(r)-\frac{\gamma}{2} \mathrm{~T}^{2}(r)-\ln \mathrm{I}_{0}(r) & -\ln 2 \pi .
\end{aligned}
$$

The graphs of $W(r)$ for several values of $\gamma$ are presented in Figure 4. Expressing $\mathcal{F}_{\mathrm{o}}$ as a function of $\boldsymbol{n}$ we get

$$
\begin{array}{r}
\mathcal{F}_{\mathrm{o}}[\varrho]=V(n):=W(r(n)) \\
=n \mathrm{~A}(n)-\frac{\gamma n^{2}}{2}-\ln \mathrm{I}_{0}(\mathrm{~A}(n))-\ln 2 \pi .
\end{array}
$$

A few graphs of $V(n)$ are presented in Figure 5. Observe that when $\gamma \geq 2$ existence of nematic ordering is manifested through appearance of potential wells in $W(r)$ and $V(n)$, characteristic for Landau-type theories of second-order phase transitions.

The total free energy (21) may now be written as

$$
\mathcal{E}[\boldsymbol{n}]=\int_{\Omega}\left\{\frac{\kappa}{2}|\nabla \boldsymbol{n}|^{2}+V(n)\right\} \mathrm{d} \boldsymbol{x} .
$$

Since

$$
|\nabla \boldsymbol{n}|^{2}=\left|\mathrm{T}^{\prime}(r) \nabla r\right|^{2}+4|\mathrm{~T}(r) \nabla \psi|^{2},
$$

this is equivalent to

$$
=\int_{\Omega}\left\{\frac{\kappa}{2}\left|\mathrm{~T}^{\prime}(r) \nabla r\right|^{2}+2 \kappa|\mathrm{T}(r) \nabla \psi|^{2}+W(r)\right\} \mathrm{d} \boldsymbol{x} .
$$

One can immediately recognize a similarity between (37) and the canonical Ginzburg-Landau energy for which $V(n)=\left(1-n^{2}\right)^{2}$. Our potential (36) is more complicated, however it maintains the same doublewell structure (when $\gamma>2$ ). In the limit as $\gamma \nearrow \infty$

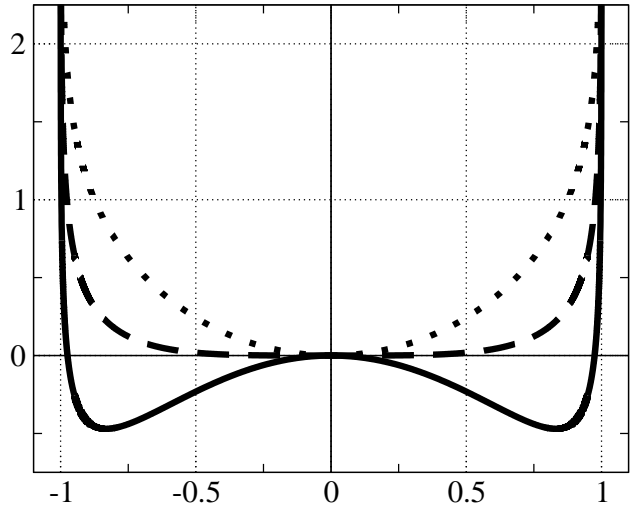

Fig. 5. Graphs of $V(n)$ given by (36). The dashed line corresponds to the critical value, $\gamma=2$; the dotted line - to $\gamma=0.2 ;$ the solid line - to $\gamma=4$.

the functional (21) is, in fact, a different regularization of Dirichlet energy for harmonic mapping from $\Omega$ into a unit circle. This connection requires a more detailed consideration and will be studied elsewhere.

\subsection{A few special solutions}

Let us now proceed with analysis of EulerLagrange equations for the free energy functional (21). Since we have shown equivalence of critical points of (21) and (37), we will actually study the latter functional (employing methods associated with Ginzburg-Landau equations). A straightforward derivation produces the following equation

$$
\kappa \Delta \boldsymbol{n}=\frac{\boldsymbol{n}}{n} V^{\prime}(n)=\frac{\boldsymbol{n}}{n} \mathrm{~A}(n)-\gamma \boldsymbol{n} .
$$

Representing (note that $\phi$ below is different from $\psi$ introduced in (31) by a factor of two)

$$
\boldsymbol{n}=n \mathrm{e}^{\mathrm{i} \phi},
$$

we may rewrite (40) as a system

$$
\begin{aligned}
\kappa \Delta n+\gamma n & =\kappa n|\nabla \phi|^{2}+\mathrm{A}(n), \\
2 \nabla n \cdot \nabla \phi+n \Delta \phi & =0 .
\end{aligned}
$$

Equation (40), or (42) and (43), has to be solved within the spatial domain $\Omega$ and should be complimented by the boundary conditions arising from the appropriate boundary terms in the total free energy functional. In what follows we consider the boundary conditions of Neumann type (26), and simplified Dirichlet boundary conditions on $\operatorname{Arg} \boldsymbol{n}(\boldsymbol{x})$. We generally assume that $\gamma>2(\gamma \leq 2$ corresponds to isotropic state in which case $V(n)$ does not have a double-well shape and thus the structure of solutions is trivial). 
A one-dimensional domain Let the physical set up be such that the system is translation-invariant in all but one spatial dimensions. Effectively we get a one-dimensional domain, $\Omega=(0, L)$. In this case equation (43) conceals a conserved quantity,

$$
\alpha=n^{2} \phi_{x}
$$

and may be integrated. Thus we may express $\phi$ through $n$ :

$$
\phi(x)=\phi(0)+\alpha \int_{0}^{x} \frac{\mathrm{d} y}{n^{2}(y)} .
$$

(The integral may not diverge since this would yield infinite variation of $\phi$ and divergence of energy.) The value of $\alpha$ needs to be determined using the boundary data. Substituting back into (42) we obtain a single equation for $n(x)$ :

$$
\kappa n_{x x}=\mathrm{A}(n)-\gamma n+\frac{\kappa \alpha^{2}}{n^{3}} .
$$

Now let us consider a few specific boundary data. In the simplest case one may prescribe the values for $\phi=\operatorname{Arg} \boldsymbol{n}, \phi(0)$ and $\phi(L)$. Since $\left|\boldsymbol{n}_{x}\right|^{2}=n_{x}^{2}+n^{2} \phi_{x}^{2}$ and $\phi_{x}=\alpha / n^{2}$, we may rewrite the total energy (for critical points) as

$$
\begin{aligned}
\mathcal{E}[\boldsymbol{n}]=\int_{0}^{L}\left\{\frac{\kappa}{2} n_{x}^{2}+V(n)\right\} \mathrm{d} x & \\
& +\frac{\kappa \delta \varphi^{2}}{2}\left[\int_{0}^{L} \frac{\mathrm{d} x}{n^{2}}\right]^{-1},
\end{aligned}
$$

where $\delta \varphi=\phi(L)-\phi(0)$ (up to a possible factor of $2 \pi k)$. Since for this kind of boundary conditions $\delta \varphi$ is fixed, the minimizers are necessarily constants. Indeed, minimizing the last two terms independently of the Dirichlet term we obtain $n(x) \equiv$ const. However this also minimizes the Dirichlet term, so the whole energy is also minimized by a constant function. Equation (46) then becomes

$$
n\left[\gamma-\kappa\left(\frac{\delta \varphi}{L}\right)^{2}\right]=\mathrm{A}(n) .
$$

So essentially this leads to renormalization of concentration $\gamma$. If $\delta \varphi<L \sqrt{(\gamma-2) / \kappa}$, (48) has a nontrivial solution, $n=n_{c}>0$, and the system is in a (chiral) nematic phase: $\phi(x)=\varphi(0)+\delta \varphi x / L$. Otherwise the only solution of (48) is $n \equiv 0$, and the system is in a uniformly isotropic state.

More interesting solutions may be obtained if we use the boundary data arising from the boundary terms in the total free energy, e.g., (26). In the onedimensional case this corresponds to

$$
\kappa \boldsymbol{n}_{x}(0)=-\boldsymbol{u}_{0}, \quad \kappa \boldsymbol{n}_{x}(L)=\boldsymbol{u}_{L},
$$

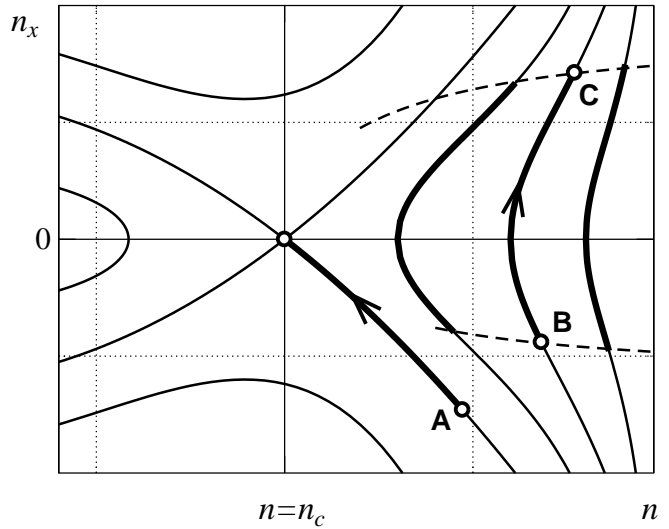

Fig. 6. Schematic representation of the phase portrait of (46) near the equilibrium point $n=n_{c}$. The fixed point itself is the solution when the boundary conditions are imposed on $\operatorname{Arg} \boldsymbol{n}$. Solutions to more complicated cases are displayed using the thick lines. For half-line, $L=\infty$, the solution is given by a portion of separatrix with endpoint (A) satisfying $\kappa n_{x}=-u_{0}$. For finite $L$, solution for a prescribed $\alpha$ is given by an orbit whose endpoints (B,C) satisfy the boundary data (50) and the "time" of travel from B to C is exactly $L$.

which may be converted into

$$
\begin{aligned}
\kappa^{2} \alpha^{2} & =n^{2}\left(u^{2}-\kappa^{2} n_{x}^{2}\right), \\
\phi & =\mp \operatorname{Arg} \boldsymbol{u}-\arccos \frac{\kappa n_{x}}{u}, \quad x=0, L .
\end{aligned}
$$

In this formulation $\alpha$ is an unknown constant to be determined so that (46) and (45) may be solved subject to (51).

Consider a semi-infinite domain, $L=\infty$ (the boundary data is only given at $x=0$ ). From the energy standpoint we immediately get that $\phi_{x} \rightarrow 0$ as $x \nearrow \infty$ and thus $\alpha=0$ (otherwise the energy is not optimal). Thus we get

$$
\phi(x) \equiv \operatorname{Arg} \boldsymbol{u}_{0},
$$

while $n(x)$ corresponds to the separatrix of (46), see Figure 6, and may be found inverting

$$
x=\frac{\kappa}{2} \int_{n_{c}}^{n}\left[V(p)-V\left(n_{c}\right)\right]^{-1 / 2} \mathrm{~d} p .
$$

(Unfortunately, no closed analytical expression for this integral may be found.)

When $L$ is finite, finding the value of $\alpha$ is a nontrivial "eigenvalue" problem: for any fixed $\alpha$ we can solve the boundary-value problem (50) for (46), see Figure 6. Using (51) we then may find $\phi(0)$ and, integrating (45), $\phi(L)$. This value, however, will only satisfy the boundary condition (51) at $x=L$ for some particular $\alpha$ (the "eigenvalue"). 
Vortex solution One of the most important special solutions for two-dimensional spatial domains is a vortex solution. Let us set

$$
\phi(\boldsymbol{x})=\arctan \left(x_{1} / x_{2}\right)
$$

and demand that $n(\boldsymbol{x})$ be a radial function with $n(0)=0$. Equation (43) for $\phi(\boldsymbol{x})$ is immediately satisfied, whereas equation (42) for $n(\boldsymbol{x})$ becomes

$$
n^{\prime \prime}+\frac{1}{z} n^{\prime}+\left(1-\frac{1}{z^{2}}\right) n=\frac{1}{\gamma} \mathrm{A}(n),
$$

where we introduced $z:=\sqrt{\gamma / \kappa} x$. The linear part of (55) is Bessel equation, however the nonlinearity on the right-hand side does not allow us to obtain explicit solutions. Still, a qualitative analysis is possible. As $z \nearrow \infty$, the $O(1)$ terms on the rightand left-hand sides have to be balanced, thus we get $n(z) \rightarrow n_{0}$, where $n_{0}$ solves

$$
\gamma n_{0}=\mathrm{A}\left(n_{0}\right) \text {. }
$$

This is an analogue of (18) with $n_{0}$ corresponding to the nematic state (remember, a nonzero solution exists if $\gamma>2$ ). So, far away from the origin the solution describes nematic phase whose director is orthogonal to the ray from the origin. The nextorder correction may be found in a straightforward manner and we get (using the original variable, $\boldsymbol{x}$ ) that as $x \nearrow \infty$,

$$
n(\boldsymbol{x})=n_{0}\left[1-\frac{\kappa}{\mathrm{A}^{\prime}\left(n_{0}\right)-\gamma} \frac{1}{x^{2}}\right]+O\left(x^{-4}\right)
$$

It is not as trivial to obtain asymptotics for $n(\boldsymbol{x})$ as $x \searrow 0$. Since $\mathrm{A}(n) \sim 2 n$ as $n \searrow 0$, in the neighborhood of zero (55) is equivalent to

$$
n^{\prime \prime}+\frac{1}{z} n^{\prime}+\left(1-\frac{2}{\gamma}-\frac{1}{z^{2}}\right) n=0 .
$$

Thus the required solution behaves as

$$
n(z) \sim C \mathrm{~J}_{1}(\sqrt{(\gamma-2) / \gamma} z) .
$$

$\left(\mathrm{J}_{1}\right.$ denotes Bessel function of the first order.) Finding the constant $C$, however, amounts to solving a nonlinear "eigenvalue" problem and cannot be accomplished analytically. Indeed, trying to solve (55) with initial data $n(0)=0, n^{\prime}(0)=c$, one finds that for small values of $c$, the solution behaves like (59) for all $z$, whereas for $c$ large, it reaches $n=1$ while $z$ is still finite. There exists precisely one value of $c$ (and the corresponding value of $C$ ) at which $n$ has the required behavior (57) at infinity. Numerical solution of (55) is presented in Figure 7.

We see that near the origin the nematic phase "melts" allowing the director to rotate. The width of

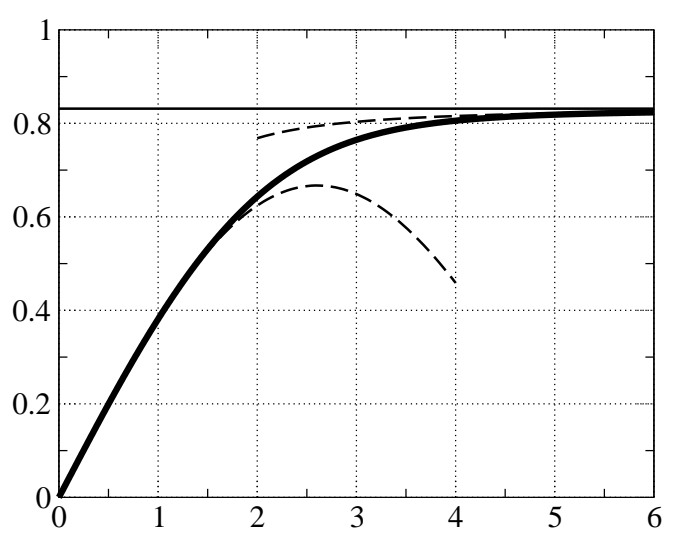

Fig. 7. Profile $n(z)$ of the vortex solution for $\gamma=4$ (the corresponding equilibrium value $n_{0} \approx 0.83$ ). The dashed lines represent approximate asymptotic solutions given by (57) and (59), and approximate numerical value for the constant $C$ in (59) is 0.86 .

the melted layer is proportional to $\sqrt{\kappa}$ and roughly to $1 / \sqrt{\gamma}$ as $\gamma \nearrow \infty$ (dependence on $\gamma$ is less trivial when $\gamma \approx 2$ because of isotropic-nematic phase transition at $\gamma=2$ ). Unlike the $\gamma \nearrow \infty$ limit, the limit as $\kappa \searrow 0$ is not particulary interesting, since it simply removes all spatial interactions. In the former case the vortex solution becomes the "true vortex,"

$$
\boldsymbol{n}(\boldsymbol{x})=\frac{\mathrm{i} \boldsymbol{x}}{x}
$$

(here we treat $\boldsymbol{x}$ as a complex variable, $x_{1}+\mathrm{i} x_{2}$ ). Generally, if we fix a finite domain $\Omega$ and impose the boundary conditions such that the argument of $\boldsymbol{n}(\boldsymbol{x})$ runs around $[0,2 \pi) k$ times, the minimizer will contain $k$ vortices. The problem of finding their precise locations is quite nontrivial and is closely related to similar problems arising in various models describing pattern formation $[19,20]$.

\section{Discussion}

In conclusion let us discuss a few ideas, generalizations, and promising directions for subsequent research within the outlined theory. The first immediate task is analysis of the full three-dimensional model instead of the simplified one considered in Section $3\left(s \in \mathbb{S}^{2}\right.$ rather than $\left.s \in \mathbb{S}^{1}\right)$. The mathematical apparatus employed here for the $\mathbb{S}^{1}$ case carries over to $\mathbb{S}^{2}$-model with little effort, and an analogue of (30) may be easily derived. The following analysis, however, is more technical due to the fact that one must deal with spherical harmonics (basis on $\mathbb{S}^{2}$ ) instead of the usual cosine functions (basis on 
$\left.\mathbb{S}^{1}\right)$. This analysis will be carried out in a follow-up to this paper [18].

\subsection{More on elasticity and nonlocal interactions}

A complete and rigorous microscopic derivation of elastic terms such as $\mathcal{F}_{\mathrm{e}}[\varrho]$ in our model, or more general, nonlocal terms (involving spatial gradients or values of various quantities at different spatial locations) in free energy functionals for liquid crystalline systems has not been accomplished yet. On phenomenological level elastic-like terms of OseenFrank and Landau-de Gennes theories are quite satisfactory and are commonly used in contemporary research. The microscopic density-functional theories may be appropriate for a better understanding of elasticity of liquid crystals, however, most of the studies in this direction are primarily concerned with expressing the phenomenological constants (e.g. elastic moduli $\kappa_{\alpha \beta}$, or constants in Landau-de Gennes theory) by means of direct pair-correlation function rather than with an explicit derivation of the functional form of nonlocal terms.

From mathematical standpoint there exist two common types of nonlocal terms in free energy functionals - elastic-like terms involving spatial gradients of various quantities at the same spatial location, e.g., (dropping differentials for compactness)

$$
\int_{\Omega} \int_{\mathbb{S}^{2}} F\left(\varrho(\boldsymbol{x}, \boldsymbol{s}), \nabla_{\Omega} \varrho(\boldsymbol{x}, \boldsymbol{s})\right) ;
$$

and "truly nonlocal" terms involving values of the fields at different points, e.g.,

$$
\iint_{\Omega} \iint_{\mathbb{S}^{2}} V\left(\boldsymbol{x}, \boldsymbol{x}^{\prime} ; \boldsymbol{s}, \boldsymbol{s}^{\prime}\right) \varrho(\boldsymbol{x}, \boldsymbol{s}) \varrho\left(\boldsymbol{x}^{\prime}, \boldsymbol{s}^{\prime}\right) .
$$

Quite often, however, there is little difference between them. For example, $F\left(\varrho, \nabla_{\Omega} \varrho\right)=\left|\nabla_{\Omega} \varrho\right|^{2}$ is equivalent to $V\left(\boldsymbol{x}, \boldsymbol{x}^{\prime} ; \boldsymbol{s}, \boldsymbol{s}^{\prime}\right)=-\delta_{\boldsymbol{s}}\left(\boldsymbol{s}^{\prime}\right) \Delta_{\Omega} \delta\left(\boldsymbol{x}-\boldsymbol{x}^{\prime}\right)$. From the microscopic physical standpoint only the latter type of interactions (62) is directly justifiable ( $n$-tuple integrals appear in various cluster expansions and correspond to $n$-particle effects). The gradient terms of the first type appear when one makes a transition from microscopic to macroscopic scale, i.e, some kind of asymptotic limit has been taken in the process. For example, if in such a limit $V\left(\boldsymbol{x}, \boldsymbol{x}^{\prime} ; \boldsymbol{s}, \boldsymbol{s}^{\prime}\right)$ converges in the sense of distributions to $-V_{\alpha \beta}\left(\boldsymbol{s}, \boldsymbol{s}^{\prime}\right) \partial_{\alpha \beta}^{2} \delta\left(\boldsymbol{x}-\boldsymbol{x}^{\prime}\right)$ (summing over $\alpha, \beta=$ $1,2,3)$, we obtain a more general form of elastic energy density. Skipping the field of director, $\boldsymbol{n}(\boldsymbol{x})$, and expressing $\mathcal{F}_{\mathrm{e}}$ directly as a functional of $\varrho(\boldsymbol{x}, \boldsymbol{s})$, we get

$$
\mathcal{F}_{\mathrm{e}}[\varrho]=\iint_{\mathbb{S}^{2}} V_{\alpha \beta}\left(s, s^{\prime}\right) \partial_{\alpha} \varrho(\boldsymbol{x}, s) \partial_{\beta} \varrho\left(\boldsymbol{x}, \boldsymbol{s}^{\prime}\right) .
$$

Observe that (11) is a particular case of (63) corresponding to a specific choice of potentials $V_{\alpha \beta}\left(s, s^{\prime}\right)$. In general, varying this potential provides additional freedom for modeling of elastic interactions.

Finally, it is important to understand that all the terms in free energies suggested here have been at most quadratic with respect to $\varrho(\boldsymbol{x}, \boldsymbol{s})$. This essentially means that we have only taken into account the two-particle interactions. This may not be sufficient for quantitative description of systems with high concentration of liquid crystalline molecules and terms cubic or of the higher order in $\varrho$ (or more complex joint probability density functions) must be present to address such cases.

\subsection{A few comments on cholesterics}

Cholesteric liquid crystals possess the so called chirality - their director rotates in space with a characteristic length-scale (the pitch) even when the system is in equilibrium and has no external constraints. The microscopic reasons for this behavior are not completely understood, however, it is clear that this phenomenon is related to the fact that the molecules of such liquid crystals possess less symmetry than simple rods. Mathematically this means that the space of orientation parameter has to be extended from $\mathbb{S}^{2}$ to a more complicated manifold. Since orientations of a generic molecule may be parameterized by rotations in a threedimensional space, the simplest extension would be to SO3 (group of rotations). A necessary step in extending our approach to systems of this kind is derivation of a proper interaction potential $U\left(s, s^{\prime}\right)$ for the Onsager-type functional in case $s, s^{\prime} \in \mathrm{SO}$. Unfortunately, we are not aware of any progress in this direction.

\subsection{Dynamics}

Study of critical points of free energy functionals provides information about equilibrium states of liquid crystalline systems. In order to explore their properties in out-of-equilibrium conditions one has to look into associated dynamics. A rigorous derivation of dynamic equations has not yet been attained 
and is related to the problem of closure of various BBGKY-type hierarchies of kinetic equations. It is quite common (and often goes along with some closure assumption) to consider dissipative gradient flow dynamics on the free energy landscape (more complex models may be obtained by coupling such dynamics with hydrodynamic equations, see [21] for a recent review). For example, the classical DoiSmoluchowski (diffusive transport) dynamics [22] for Onsager free energy functionals is governed by

$$
\partial_{t} \varrho(s, t)=\nabla_{\mathbb{S}^{2}} \cdot\left\{\varrho(s) \nabla_{\mathbb{S}^{2}} \frac{\delta \mathcal{F}_{\text {Ons }}[\varrho]}{\delta \varrho(s)}\right\} .
$$

This is the gradient flow in the so called Wasserstein2 metric [16] of probability measures. (Note that here the probability density of orientations, $\varrho(s)$, is $\boldsymbol{x}$-independent and the divergence and gradient operators are native to the sphere $\mathbb{S}^{2}$.) At the same time dynamics for theories based on the order parameter, e.g, Landau-de Gennes theory, is described by the usual gradient flow (in $\mathbb{L}^{2}$ metric)

$$
\partial_{t} \mathbf{O}(\boldsymbol{x}, t)=-\frac{\delta \mathcal{E}_{\mathrm{LdG}}[\mathbf{O}]}{\delta \mathbf{O}(\boldsymbol{x})} .
$$

As demonstrated in the previous sections, within the framework of our theory there is a direct relation between the order parameter field and $\boldsymbol{x}$-dependent orientation density. Thus this theory provides a natural setting for studying the question of whether it is possible to relate the Doi-Smoluchowski-type dynamics for $\varrho(\boldsymbol{x}, \boldsymbol{s})$ with Landau-type dynamics of the order parameter fields. (The gradient flow equation for evolution of $\varrho(\boldsymbol{x}, \boldsymbol{s})$ would be identical to (64) except the gradient and divergence operators would then act in the full space $\Omega \times \mathbb{S}^{2}$ and the full free energy (10) would replace $\mathcal{F}_{\text {Ons }}[\varrho]$.)

\section{Acknowledgements}

The authors thank referees for valuable comments and references. V.S. is partially supported by Nuffield Foundation Grant NAL32562.

\section{References}

[1] C. Oseen. Theory of liquid crystals. Transactions of Faraday Society, 29:883-899, 1933.

[2] F. C. Frank. On the theory of liquid crystals. Discussions of Faraday Society, 25:19-28, 1958.

[3] P. G. de Gennes and J. Prost. The physics of liquid crystals. Clarendon Press, Oxford, 1995.
[4] L. Onsager. The effects of shape on the interaction of colloidal particles. Annals of New York Academy of Sciences, 51:627-659, 1949.

[5] Y. Singh. Molecular theory of liquid crystals: Applications to the nematic phase. Physical Review A, 30(1):583-593, 1984.

[6] S. Singh. Phase transitions in liquid crystals. Physics Reports, 324(2):107-269, 2000.

[7] I. Fatkullin and V. Slastikov. A note on the Onsager model of nematic phase transitions. Communications in Mathematical Sciences, 3(1):21-26, 2005.

[8] I. Fatkullin and V. Slastikov. Critical points of the Onsager functional on a sphere. Nonlinearity, 18:2562$2580,2005$.

[9] M. J. Stephen and J. P. Straley. Physics of liquid crystals. Reviews of Modern Physics, 46(4):617-704, 1974.

[10] G. J. Vroege and N. H. W. Lekkerkerker. Phase transitions in lyotropic colloidal and polymer liquid crystals. Reports on Progress in Physics, 55:1241-1309, 1992.

[11] M. P. Allen. Liquid crystal systems. Computational Soft Matter: From Synthetic Polymers to Proteins, Lecture Notes, 23:289-320, 2004.

[12] S. Chandrasekhar. Liquid crystals. Cambridge University Press (2nd edition), Cambridge, 1993.

[13] R. G. Priest. Theory of the Frank elastic constants of nematic liquid crystals. Physical Review A, 7(2):720729, 1973.

[14] J. P. Straley. Frank elastic constants of the hard-rod liquid crystal. Physical Review A, 8(4):2181-2183, 1973.

[15] W. Maier and A. Saupe. Eine einfache molekulare Theorie des nematischen kristallinflüssingen Zustandes. Zeitschrift für Naturforschung, 13:564, 1958.

[16] L Ambrosio, N. Gigli, and G. Savaré. Gradient flows in metric spaces and in the space of probability measures. Lectures in Mathematics, Birkhäuser Verlag, BaselBoston-Berlin, 2005.

[17] L. Longa, D. Monselesan, and H.-R. Trebin. An extension of the Landau-Ginzburg-de Gennes theory for liquid crystals. Liquid Crystals, 2(6):769-796, 1987.

[18] I. Fatkullin and V. Slastikov. On Spatial variations of nematic ordering. Part II. In preparation.

[19] M. C. Cross and P. C. Hohenberg. Pattern formation outside of equilibrium. Reviews of Modern Physics, 65:851-1112, 1993.

[20] F. Bethuel, H. Brezis, and F. Helein. Ginzburg-Landau vortices. Progress in nonlinear differential equations and their applications, Birkhäuser, Boston, 13, 1994.

[21] M. Kröger. Simple models for complex nonequilibrium fluids. Physics Reports, 390:453-551, 2004.

[22] M. Doi. Molecular dynamics and rheological properties of concentrated solutions of rodlike polymers in isotropic and liquid crystalline phases. Journal of Polymer Science. Polymer Physics Edition, 19:229-243, 1981. 